\title{
Interference Management without CSIT: A Broadcast Approach
}

\author{
Maha Zohdy and Ali Tajer \\ Rensselaer Polytechnic Institute
}

\author{
Shlomo Shamai (Shitz) \\ Technion Israel Institute of Technology
}

\begin{abstract}
Effective interference management in the multiuser interference channel strongly hinges on the availability of the channel state information at the transmitters (CSIT). In a broad range of emerging large-scale and distributed networks (e.g., the Internet of Things), however, acquiring the CSIT is prohibitive, due to the extensive information exchange that it imposes. In such circumstances, as a result, the interference management approaches that rely on the CSIT lose their effectiveness. This paper focuses on the two-user interference channel, and proposes a broadcast approach to interference management. Its hallmark is that the transmitters, unlike the receivers, are completely oblivious to instantaneous channel states. Each transmitter splits its message into multiple superimposed encoded information layers, where each layer is adapted to a given possible state for the combined states of all channels. Depending on the relative strengths of the direct and interfering channels, each receiver opportunistically decodes a subset of the received layers from both transmitters. An average achievable rate region is delineated serving as an inner bound on the average capacity region of the Gaussian interference channel in the absence of CSIT. Finally, it characterizes the gap between the achievable average sum-rate and the sum-rate capacity with the full CSIT in the asymptote of high signal-to-noise ratio. Numerical evaluations show that the cost of lacking CSIT is often insignificant.
\end{abstract}

\section{INTRODUCTION}

The multiuser interference channel constitutes a canonical building block in interference-limited wireless networks in which multiple transmitters communicate with their designated receivers, while interfering with others. Designing and analyzing interference management schemes has a rich literature. Irrespective of their discrepancies, the existing approaches often rely on the accurate availability of the channel state information at the transmitters (CSIT) as well as the receivers. While acquiring such information at the receiver can be facilitated through training sessions, acquiring it at the transmitters necessitates each receiver reporting it to all transmitters. Such additional information exchange incurs excessive communication costs and latency, especially when the network size grows.

Under the ideal assumption of perfect availability of the CSIT at all transmitters, some representative known results include the achievable rate region due to Han-Kobayashi (HK) [1], [2], which is shown to achieve rates within one bit of the capacity region for the Gaussian interference channel [3]. While unknown in its general form, the capacity region is known in special cases, including the strong interference channel [4] and [5], the discrete additive degraded interference

The work of M. Zohdy and A. Tajer was supported in part by the U. S. National Science Foundation under the CAREER Award ECCS-1554482, and the grant ECCS-1933107. The work of S. Shamai (Shitz) has been supported by the European Union's Horizon 2020 Research And Innovation Programme, grant agreement no. 694630 . channel [6], and certain classes of the deterministic interference channel [7]-[10]. When channels undergo fast variations, interference alignment achieves the sum-rate capacity in the asymptote of high signal-to-noise ratio (SNR) [11] and [12].

An effective approach to circumventing the lack of the CSIT is the broadcast approach, initially proposed for the compound broadcast channel [13]. Based on that, a broadcast strategy was introduced in [14] and [15] for the slowly-fading single-user channel. In the broadcast approach, a transmitter, that is oblivious to the channel linking it to its designated receiver, performs superposition coding by splitting its data stream into a number of independently-generated coded layers with different rates. The rate of each layer is adapted to a specific channel state. The transmitter then superimposes and transmits all the generated layers and the receiver decodes as many layers as the actual quality of the channel affords. This approach is then studied under various assumptions for multiple access communications [16]-[21], and for interference channels in [22]-[24]. Specifically, the studies in [22] and [24] adopt superposition coding for opportunistic transmission and decoding of interference, and provide the analysis in the high-SNR regime (generalized degrees of freedom). The study in [23] investigates opportunistic decoding of the interference, with the emphasis on the decoding complexity and outage analysis. Unlike these studies, in this paper we focus on the non-asymptotic regime and characterize an average achievable rate region.

In this paper, we focus on the two-user slowly fading Gaussian interference channel with the channel state information only known to the receivers. We propose a broadcast transmission approach to circumvent the lack of CSIT. In the absence of the CSIT, designing an effective broadcast approach consists in balancing the strengths of the intended and the interfering signals at each receiver. This, in turn, fundamentally rests on proper rate-splitting at each transmitter such that there are sufficient independent information layers at each transmitter so that we can designate each information layer to one network state (combined channels states). The key distinction between the proposed approach for the interference channel and the counterpart approaches for the single-user channel is that in the single-user channel, the transmitter adapts its information layers to the direct channel linking it to the receiver. This idea is directly adopted for the multiple access channel as well [18]. In the proposed approach, on the other hand, the information layers at each transmitter are adapted not only to the direct channels, but also to the interfering channels, rendering the information layers being adapted to different combinations of channel states. We remark that as opposed 
to [24], which focuses on the high SNR performance, we focus on the non-asymptotic regime and characterize an achievable rate region.

We start by formalizing the model and describing the superposition coding strategy and the decoding schemes. Then we delineate the associated average achievable rate region under a simple successive decoding scheme, which can be fully characterized by optimizing the power allocation between the transmitted layers. For the special case of symmetric channels, we determine the power allocation required to equalize the rates of each codebook that is decoded by the receivers in different channel states. Based on such power allocation, we compare the resulting average achievable sum-rate to that of the HK scheme with perfect CSIT and characterize the gap in the high SNR regime.

\section{Channel Model}

Consider the two-user Gaussian interference channel. The coefficient of the channel connecting transmitter $j$ to receiver $i$ is denoted by $h_{i j}^{\prime}$ for $i, j \in\{1,2\}$. We refer to $h_{i i}^{\prime}$ and $h_{i j}^{\prime}$ as the direct and cross channel coefficients, respectively, $\forall i \neq j$. Each channel is assumed to follow a block fading model in which the channel coefficients remain constant for the duration of a transmission block of $n$ channel uses, and randomly change to another state afterwards. We consider an $\ell$-state channel model in which each channel coefficient randomly and independently encounters one of the $\ell$ states $\left\{\alpha_{1}, \ldots, \alpha_{\ell}\right\}$. Without loss of generality, we assume that $0<\alpha_{1}<\cdots<\alpha_{\ell}<+\infty$. The signal received by receiver $i$ is given by

$$
Y_{i}^{\prime}=h_{i i}^{\prime} X_{i}^{\prime}+h_{i j}^{\prime} X_{j}^{\prime}+N_{i}^{\prime},
$$

where $X_{i}^{\prime}$ and $Y_{i}^{\prime}$ denote the symbols transmitted and received by transmitter and receiver $i$, respectively, and $N_{i}^{\prime} \sim \mathcal{N}\left(0, \sigma^{2}\right)$ accounts for the additive Gaussian noise with zero-mean and variance $\sigma^{2}$. The transmitted symbol $X_{i}^{\prime}$ is subject to an average power constraint $P_{i}^{\prime}$, i.e., $\mathbb{E}\left[\left|X_{i}^{\prime}\right|^{2}\right] \leq P_{i}^{\prime}$. The $\ell$ state interference channel in (1) gives rise to an interference network with $\ell^{4}$ different states. The instantaneous network state is assumed to be fully known at both receivers, while being unknown to the transmitters. A statistically equivalent form of the $\ell$-state interference channel in (1) is the standard interference channel model given by [25] and [26]

$$
Y_{i}=X_{i}+h_{i j} X_{j}+N_{i}
$$

where the channel coefficient $h_{i j}$ is given by

$$
h_{i j} \triangleq \frac{h^{\prime}{ }_{i j}}{h^{\prime}{ }_{i i}},
$$

and the transmitted symbols, the noise power, and the transmission power are normalized as

$$
X_{i} \triangleq \frac{h^{\prime}{ }_{i i}}{\sigma} \cdot X_{i}^{\prime}, \quad N_{i} \triangleq \frac{N_{i}^{\prime}}{\sigma}, \quad \text { and } \quad P_{i}=\left(\frac{h^{\prime}{ }_{i i}}{\sigma}\right)^{2} \cdot P_{i}^{\prime},
$$

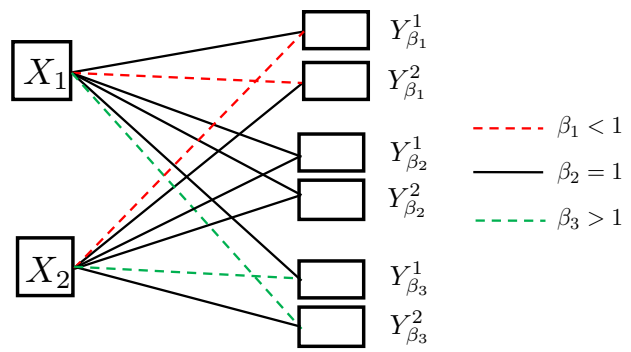

Fig. 1: Equivalent broadcast network for the two-state $(\ell=2)$ Gaussian interference channel.

respectively. Consequently, the normalized Gaussian noise $N_{i}$ is distributed as $\mathcal{N}(0,1)$. Corresponding to all possible combinations of the $\ell$-state interference channel in (1), by invoking the normalization in (3) it can be readily verified that the cross channel gain $h_{i j}^{2}$ undergoes one of the $K \triangleq \ell(\ell-1)+1$ possible states denoted by $\left\{\beta_{1}, \ldots, \beta_{K}\right\}$, where $0<\beta_{1}<\cdots<\beta_{K}<+\infty$. Since the direct channel gains in (2) is normalized to 1 , the network state is specified by the combination of the two cross links, rendering $K^{2}$ states for the network. Specifically, for the two-state channel, the cross channel gain takes one of the three states $\beta_{1} \triangleq \frac{\alpha_{1}}{\alpha_{2}}, \beta_{2} \triangleq \frac{\alpha_{1}}{\alpha_{1}}=\frac{\alpha_{2}}{\alpha_{2}}=1$, and $\beta_{3} \triangleq \frac{\alpha_{2}}{\alpha_{1}}$.

The network is in state $\left(\beta_{s}, \beta_{t}\right)$ when $\left(h_{12}^{2}, h_{21}^{2}\right)=\left(\beta_{s}, \beta_{t}\right)$. Accordingly, when the network is in state $\left(\beta_{s}, \beta_{t}\right)$, we denote the received signals at the two receivers by

$$
Y_{\beta_{s}}^{i}=X_{i}+\sqrt{\beta_{s}} X_{j}+N_{i} .
$$

Hence, when adopting a broadcast approach to transmission, this network can be equivalently presented as a network with two transmitters and $K^{2}$ receiver pairs, where each receiver pair corresponds to one possible channel state. In case of a symmetric interference channel we have $h_{21}^{2}=h_{12}^{2}$, and the number of possible channel combinations reduces to $K$, rending an equivalent network with two transmitters and $2 K$ receivers. Figure 1 depicts such a network for $\ell=2$.

Finally, we define $q_{k}^{i}$ as the probability that the channel gain $h_{i j}^{2}$ is in the state $\beta_{k}$. In this paper, we will be focusing on the two-state two-user Gaussian interference channel and will be characterizing an achievable rate region for the multiuser network specified by (5). Throughout the paper, we use the notion $C(x, y) \triangleq \frac{1}{2} \log _{2}\left(1+\frac{x}{1+y}\right)$ and use the shorthand notation $\left\{x_{i}\right\}_{i=j}^{k}$ to denote the sequence $\left\{x_{j}, \ldots, x_{k}\right\}$.

\section{RATE-SPlitTing And Decoding Scheme}

\section{A. Overview of Broadcast Approach}

According to the proposed approach devised for the twostate single-user channel with the states $\alpha_{1}$ and $\alpha_{2}$, transmitter $i$ splits its message to two information layers, one adapted to the weak channel state $\alpha_{1}$, and one to the strong channel state $\alpha_{2}$. Each transmitter encodes its information stream by two layers and adapts the distribution of power between them according to its own channel state. Subsequently, the receiver performs sequential decoding approach. When the channel is in the weak state, only the codebook assigned to the weak 
channel is decoded, and when the channel is in the strong state, additionally the second codebook assigned to the strong channel is also decoded.

\section{B. State-adaptive Layering}

Since each transmitter has the two opposing effects on its designated and non-designated receivers, each transmitter is devised to perform rate-splitting adapted to the combined state of the network, as opposed to its direct channel to its designated receiver. In particular, transmitter $i \in\{1,2\}$ splits its message into a prescribed finite number of encoded layers, and all the layers are then superimposed and transmitted to the corresponding receiver. The rate of each layer is adapted to a particular network state, such that it can be decoded successfully at that channel state by the intended receiver.

When viewing the interference channel from the perspective of the broadcast approach, transmission of each transmitter to each of the $2 K^{2}$ receivers can be viewed as multiple access communication. The reason is that unlike the interference channel, in which each receiver views the signals from the non-designated transmitter as disruptive interference, in the broadcast approach each receiver aims to decode as many information layer as possible that are transmitted by both transmitters. Correspondingly, the multiuser network model in (5) can be viewed as a collection of multiple access channels.

A pivotal property of these multiple access channels is that, for $\beta_{l}<\beta_{k}$, the multiple access channel at receiver $Y_{\beta_{l}}^{i}$ serves as a degraded version of the channel at $Y_{\beta_{k}}^{i}$. Therefore, receiver $Y_{\beta_{k}}^{i}$ can successfully decode all the layers decoded by $Y_{\beta_{\ell}}^{i}$, in addition to the additional layers designated to the channels $\left\{\beta_{l+1}, \ldots, \beta_{k}\right\}$. At receiver $Y_{\beta_{k}}^{i}$, a layer decoded form transmitter $i$ directly increases the achievable rate of receiver $i$, whereas a layer decoded from transmitter $j$ indirectly increases the achievable rate at receiver $i$ by canceling a part of the interfering signal. Based on these observations, in the twostate channel $(\ell=2)$, transmitter $i$ splits its message into six layers denoted by $\left\{V_{k}^{i}, U_{k}^{i}\right\}_{k=1}^{3}$, where

- Layer $V_{k}^{i}$ is adapted to the cross channel state at the unintended receiver $Y_{\beta_{k}}^{j}, \forall k \in\{1,2,3\}, i \neq j$.

- Layer $U_{k}^{i}$ is adapted to the cross channel state at the intended receiver $Y_{\beta_{k}}^{i}, \forall k \in\{1,2,3\}$.

This layering approach and the correspondence between the codebooks and the channel states are illustrated in Fig. 2.

\begin{tabular}{c|c|c|c|}
\multicolumn{1}{c}{} & $\beta_{1}$ & $\beta_{2}$ & $\beta_{3}$ \\
$Y_{\beta_{i}}^{1}$ & $U_{1}^{1}$ & $U_{2}^{1}$ & $U_{3}^{1}$ \\
\cline { 2 - 4 }$Y_{\beta_{i}}^{2}$ & $V_{1}^{1}$ & $V_{2}^{1}$ & $V_{3}^{1}$ \\
\cline { 2 - 4 } & &
\end{tabular}

User 1

$$
\begin{array}{c|c|c|c|}
Y_{\beta_{i}}^{2} & \beta_{1} & \beta_{2} & \beta_{3} \\
\cline { 1 - 3 } Y_{\beta_{i}}^{1} & U_{1}^{2} & U_{2}^{2} & U_{3}^{2} \\
\cline { 2 - 4 } & \multicolumn{4}{c}{\text { User } 2} & V_{2}^{2} & V_{3}^{2} \\
\hline \multicolumn{4}{c}{\text { User }}
\end{array}
$$

Fig. 2: Layering and codebook assignment scheme $(\ell=2)$.

\section{Successive Decoding Strategy}

In this subsection, we outline the interactive roles between multiple receivers underlying the proposed opportunistic successive decoding strategy. In the broadcast interference channel in (5), each codebook will be decoded by multiple receivers. Hence, the rate of each codebook will be constrained by its associated most degraded channel state. Furthermore, any undecoded layer at a certain receiver imposes interference, which degrades the achievable rate at that receiver. Motivated by these premises, we devise a simple opportunistic successive decoding scheme. This decoding scheme, specifically, identifies (i) the set of receivers at which each layer is decoded, and (ii) the order of successive decoding at each receiver.

To formalize the decoding strategy, we denote the set of receivers that decode codebook $V_{k}^{i}$ by $\mathcal{D}_{v_{k}^{i}}$, and those that decode $U_{k}^{i}$ by $\mathcal{D}_{u_{k}^{i}}$. Next, note that transmitter $i$ adapts layers $\left\{V_{k}^{i}\right\}_{k=1}^{3}$ as baseline layers for the intended receivers $\left\{Y_{\beta_{k}}^{i}\right\}_{k=1}^{3}$, as well as layers to be decoded by the set of unintended receivers $\left\{Y_{\beta_{k}}^{j}\right\}_{k=1}^{3}$ for $i \neq j$, opportunistically. Hence, the set $\mathcal{D}_{v_{k}^{i}}$, consisting of the set of receivers at which codebook $V_{k}^{i}$ is decoded, is given by

$$
\mathcal{D}_{v_{k}^{i}}=\left\{Y_{\ell}^{i}\right\}_{\ell=1}^{3} \cup\left\{Y_{\ell}^{j}\right\}_{\ell=k}^{3}, \quad i \neq j .
$$

On the other hand, transmitter $i$ adapts the layers $\left\{U_{k}^{i}\right\}_{k=1}^{3}$ only to the intended receivers $\left\{Y_{\beta_{k}}^{i}\right\}_{k=1}^{3}$. Consequently, the set $\mathcal{D}_{u_{k}^{i}}$, consisting of the set of receivers at which codebook $U_{k}^{i}$ is decoded, is given by

$$
\mathcal{D}_{u_{k}^{i}}=\left\{Y_{\ell}^{i}\right\}_{\ell=k}^{3} .
$$

Based on (6) and (7), next, we outline the proposed successive decoding order at each of the six receivers.

- Receiver $Y_{\beta_{1}}^{i}$ : It decodes the first baseline layer from the transmitter affected by the stronger channel, namely $V_{1}^{i}$, given that the direct channel is stronger. Then, it decodes the first baseline layer of the other transmitter $V_{1}^{j}$, followed by the remaining baseline layers $\left\{V_{\ell}^{i}\right\}_{\ell=2}^{3}$ from its intended transmitter $i$. Finally, layer $U_{1}^{i}$ is decoded.

- Receiver $Y_{\beta_{2}}^{i}$ : It decodes the first baseline layers, $\left\{V_{1}^{i}, V_{1}^{j}\right\}$ (decodable by receiver $Y_{\beta_{1}}^{i}$ ), followed by $\left\{V_{2}^{i}, V_{2}^{j}\right\}$ adapted to $Y_{\beta_{2}}^{i}$ from both transmitters. Then, the remaining baseline layer $V_{3}^{i}$ from its intended transmitter is decoded in addition to layers $\left\{U_{1}^{i}, U_{2}^{i}\right\}$.

- Receiver $Y_{\beta_{3}}^{i}$ : It decodes the first baseline layer from the transmitter affected by the stronger channel, namely $V_{1}^{j}$, given that the cross channel state is stronger, followed by the corresponding baseline layer from the intended transmitter $V_{1}^{i}$. Then, it decodes layers $\left\{V_{2}^{j}, V_{2}^{i}\right\}$ (decodable by receiver $Y_{\beta_{2}}^{i}$ ). Finally, layers $\left\{V_{3}^{j}, V_{3}^{i}\right\}$ adapted to receiver $Y_{\beta_{3}}^{i}$ are decoded in addition to all the remaining layers $\left\{U_{1}^{i}, U_{2}^{i}, U_{3}^{i}\right\}$ from the intended transmitter.

Table I illustrates the successive decoding scheme.

\section{Average Achievable Rate Region}

In this section, we characterize the average achievable rate region corresponding to the adaptive layering scheme and successive decoding policy outlined in Section III. Based on the characterized average rate region, we further show that in the high SNR regime, for fixed channel coefficients, the average achievable sum-rate of the proposed scheme is within a constant gap from the sum-rate capacity with full CSIT. 
Table I: Successive decoding order at the receivers.

\begin{tabular}{|c||c|c|c|c|c|c|c|c|c|}
\hline Receiver & Stage 1 & Stage 2 & Stage 3 & Stage 4 & Stage 5 & Stage 6 & Stage 7 & Stage 8 & Stage 9 \\
\hline \hline$Y_{\beta_{1}}^{i}$ & $V_{1}^{i}$ & $V_{1}^{j}$ & $V_{2}^{i}$ & $V_{3}^{i}$ & $U_{1}^{i}$ & & & & \\
\hline$Y_{\beta_{2}}^{i}$ & $V_{1}^{i}$ & $V_{1}^{j}$ & $V_{2}^{i}$ & $V_{2}^{j}$ & $V_{3}^{i}$ & $U_{1}^{i}$ & $U_{2}^{i}$ & & \\
\hline$Y_{\beta_{3}}^{i}$ & $V_{1}^{j}$ & $V_{1}^{i}$ & $V_{2}^{j}$ & $V_{2}^{i}$ & $V_{3}^{j}$ & $V_{3}^{i}$ & $U_{1}^{i}$ & $U_{2}^{i}$ & $U_{3}^{i}$ \\
\hline
\end{tabular}

\section{A. Average Rate region}

An achievable rate region is defined as the set of achievable rates of the codebooks $\left\{V_{k}^{i}, U_{k}^{i}\right\}_{k=1}^{3}, \forall i \in\{1,2\}$. The achievable rate of codebook $V_{k}^{i}\left(U_{k}^{i}\right)$ is bounded by the minimum achievable rate at the receivers in the set $\mathcal{D}_{v_{k}^{i}}\left(\mathcal{D}_{u_{k}^{i}}\right)$, where the successive decoding order at each receiver is outlined in Table I. We denote the rates of codebooks $V_{k}^{i}$ and $U_{k}^{i}$ by $R_{v_{k}^{i}}$ and $R_{u_{k}^{i}}$, respectively. Similarly, we denote the power fractions of the total power $P_{i}$ allocated to codebooks $V_{k}^{i}$ and $U_{k}^{i}$ by $\gamma_{v_{k}^{i}}$ and $\gamma_{u_{k}^{i}}$, respectively. Finally, we define $R_{i}\left(\beta_{j}, \beta_{k}\right)$ as the total achievable rate for user $i$ in the channel state $\left(\beta_{j}, \beta_{k}\right)$ and define $\bar{R}_{i} \triangleq \mathbb{E}\left[R_{i}\left(\beta_{j}, \beta_{k}\right)\right]$ as the average achievable rate at receiver $i$, where the expectation is taken with respect to the combined channel states distribution. The average achievable rate region is formally characterized in Theorem 1 .

Theorem 1. In the two-state channel, the average achievable rate region corresponding to the layering scheme in Fig. 2 and the decoding order in Table I encloses the set of rates $\left\{R_{v_{k}^{i}}, R_{u_{k}^{i}}\right\}$ for $i \in\{1,2\}, k \in\{1,2,3\}$ that satisfy

$$
\begin{aligned}
& \bar{R}_{1} \leq \sum_{j=1}^{3} \sum_{k=1}^{3} q_{j}^{1} q_{k}^{2} R_{1}\left(\beta_{j}, \beta_{k}\right), \\
& \bar{R}_{2} \leq \sum_{j=1}^{3} \sum_{k=1}^{3} q_{j}^{1} q_{k}^{2} R_{2}\left(\beta_{j}, \beta_{k}\right),
\end{aligned}
$$

where the total achievable rates are bounded by

$$
R_{i}\left(\beta_{j}, \beta_{k}\right) \leq r_{i}(j, k),
$$

and the constants $\left\{r_{i}(j, k)\right\}$ are specified in Appendix A.

\section{B. Gap to Sum-rate Capacity}

In this subsection, we demonstrate that the proposed adaptive layering and successive decoding schemes, achieve an average sum-rate that for fixed channel gains lies within a constant gap of the sum-rate capacity of the two-user Gaussian interference channel with full CSIT in the high SNR regime. For the convenience in notations and analysis, we focus on the symmetric setting, i.e., $h_{i j}^{2}=h_{j i}^{2}=h^{2}$, and assume symmetric average power constraint $P_{1}=P_{2}=P$ as well as symmetric probability distribution, i.e., $q_{k}^{1}=q_{k}^{2}=q_{k}$. The results can be readily generalized to the non-symmetric setting as well.

In the symmetric case, $h^{2} \in\left\{\beta_{1}, \beta_{2}, \beta_{3}\right\}$, the interference channel specified by (5) randomly reduces to either a weak interference channel if $h^{2}=\beta_{1}$, or to a strong interference channel if $h^{2}=\beta_{2}$ or $h^{2}=\beta_{3}$.
In order to quantify the desired average rate gap, we analyze the gap in the weak and strong interference regimes separately, where the average of which provides the average rate gap.

- Weak interference: In this setting, in order to quantify the gap, we first quantify the gap between our average sum-rate and that of HK. Subsequently, by leveraging the known results on the gap between the sum-rate of HK and the sum-rate capacity, we delineate an upper bound on the average sum-rate gap of interest. In particular, we consider the simple scheme of [3] in which the effective power of some messages is normalized to 1 at each receiver. This simple HK scheme is known to achieve a sum-rate within 2-bits from the sum-rate capacity. We remark that in the case of weak interference, the achievable sum-rate of the HK-scheme in [3], depending on the channel parameters, is either bounded by the capacity of the multiple access channel formed at each receiver or by the rate constraint of decoding the common message at the unintended receiver. These two different regimes are identified by the relation between the power constraint $P$ and the channel gains $\beta_{k}$.

- Strong interference: For the case of strong interference channel, the sum-rate capacity with full CSIT is known, which can be found by evaluating the sum-rate of the intersection of two capacity regions corresponding to two multiple access channels formed by the transmitters and each of the two receivers [5]. We leverage this to quantify the sum-rate gap of interest.

Based on these, we delineate the gap between our average sum-rate and the sum-rate capacity in the next theorem.

Theorem 2. In the asymptote of large values of $P$, the average sum-rate achievable by the proposed broadcast approach lies within a constant gap from the average sum-rate capacity of the symmetric Gaussian interference channel with full CSIT. The gap in the sum-rate is characterized as follows:

$$
\Delta \leq \frac{1}{2} \log _{2}\left(\frac{2^{q_{2}}\left(2+\beta_{3}\right)^{1-q_{2}}}{\left(1+\beta_{1}\right)^{1-q_{1}}}\right)+q_{1} .
$$

\section{Numerical Evaluations}

In this section, we evaluate the value of knowing the CSIT. Specifically, we compare the average sum-rate of the proposed approach to that of the HK scheme. In Fig. 3, we illustrate the fraction of the average sum-rate of the HK scheme lost due to lack of the CSIT. Specifically, we illustrate the normalized gaps, that is the values of $\Delta$ in (11), normalized by the average sum-rate of the HK scheme. This figure demonstrates the variations in gap to the average sum-rate capacity by 


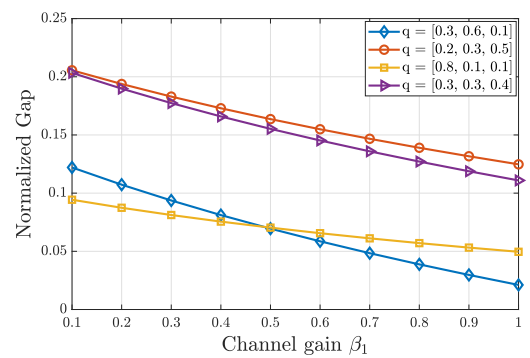

Fig. 3: Normalized sum-rate gap versus channel gain $\beta_{1}$.

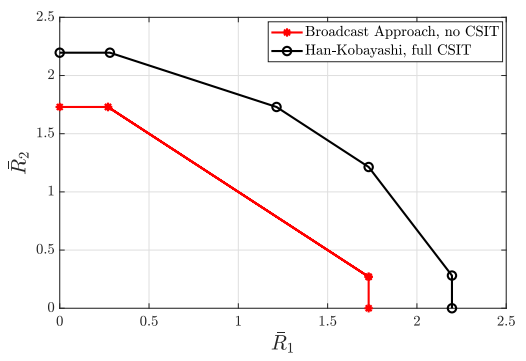

Figure 4: Average rate region for symmetric channel.

varying the weak channel gain $\beta_{1}$ in its entire range $(0,1]$ when $P=20 \mathrm{~dB}$. The main observation is that the sum-rate capacity gap due to lack of the CSIT varies between $2 \%$ to $20 \%$ in this setting.

We also evaluate the average rate region specified in Theorem 1. Figure 4 demonstrates the symmetric case where the average power constraints at transmitter 1 and 2 are $P_{1}=P_{2}=5$. The probabilities at transmitters are set to $\left[q_{1}^{i}, q_{2}^{i}, q_{3}^{i}\right]=[0.3,0.3,0.4]$, and the weak channel state at each receiver is given by $\beta_{1}=0.5$. It is observed that in despite of losing the entire CSIT, the proposed approach still recovers a considerable fraction of HK's average rate region.

\section{CONCLUSION}

In this paper, we have proposed a novel distributed interference management scheme based on the broadcast approach. We have considered the two-user Gaussian interference channel in which each transmitter is oblivious to all channel state. Based on the combined network state, a multi-layer superposition coding scheme is adopted at each transmitter combined with an opportunistic decoding scheme at the receivers. We have characterized an average achievable rate region, and have shown that in the high signal-to-noise ratio regime, the corresponding sum-rate lies within a constant gap of the sumrate capacity of the interference channel with full channel state information.

\section{APPENDIX A}

\section{CONSTANTS OF THEOREM 1}

By defining $\Gamma_{v_{\ell}^{i}} \triangleq \sum_{k=1}^{\ell} v_{k}^{i}$ and $\Gamma_{u_{\ell}^{i}}=\sum_{k=1}^{\ell} u_{k}^{i}, \forall i, j \in$ $\{1,2\}, \forall \ell \in\{1,2,3\}, i \neq j$, we have

$$
\begin{aligned}
& r_{i}(1,1) \triangleq \sum_{k=2}^{3} a_{i}\left(v_{k}^{i}\right)+a_{i}\left(u_{1}^{i}\right)+\min _{j \in\{1,2\}} a_{j}\left(v_{1}^{i}\right), \\
& r_{i}(1,2) \triangleq a_{i}\left(v_{3}^{i}\right)+a_{i}\left(u_{1}^{i}\right)+\min _{\ell, n \in\{1,2\}} a_{i}\left(v_{\ell}^{i}\right)+b_{j}\left(v_{n}^{i}\right),
\end{aligned}
$$

$$
\begin{aligned}
& r_{i}(1,3) \triangleq \sum_{k=1}^{3} a_{i}\left(v_{k}^{i}\right)+a_{i}\left(u_{1}^{i}\right), \\
& r_{i}(2,1) \triangleq \sum_{k=2}^{3} b_{i}\left(v_{k}^{i}\right)+\sum_{k=1}^{2} b_{i}\left(u_{k}^{i}\right)+\min \left\{b_{i}\left(v_{1}^{i}\right), a_{j}\left(v_{1}^{i}\right)\right\}, \\
& r_{i}(2,2) \triangleq \sum_{k=1}^{3} b_{i}\left(v_{k}^{i}\right)+\sum_{k=1}^{2} b_{i}\left(u_{k}^{i}\right), \\
& r_{i}(2,3) \triangleq \sum_{k=1}^{3} b_{i}\left(v_{k}^{i}\right)+\sum_{k=1}^{3} b_{i}\left(u_{k}^{i}\right), \\
& r_{i}(3,1) \triangleq \sum_{k=2}^{3} c_{i}\left(v_{k}^{i}\right)+\sum_{k=1}^{3} c_{i}\left(u_{k}^{i}\right)+\min \left\{c_{i}\left(v_{1}^{i}\right), a_{j}\left(v_{1}^{i}\right)\right\}, \\
& r_{i}(3,2) \triangleq \sum_{k=1}^{3} c_{i}\left(v_{k}^{i}\right)+\sum_{k=1}^{3} c_{i}\left(u_{k}^{i}\right), \\
& r_{i}(3,3) \triangleq \sum_{k=1}^{3} c_{i}\left(u_{k}^{i}\right)+\min _{n, \ell, p \in\{1,2\}} c_{n}\left(v_{1}^{i}\right)+c_{\ell}\left(v_{2}^{i}\right)+c_{p}\left(v_{3}^{i}\right),
\end{aligned}
$$

where $\left\{a_{i}(\cdot), b_{i}(\cdot), c_{i}(\cdot)\right\}, \forall i \in\{1,2\}$ are defined below

$$
\begin{aligned}
& a_{i}\left(v_{1}^{i}\right) \triangleq C\left(\gamma_{v_{1}^{i}} P_{i}, \beta_{1} P_{j}+\left(1-\gamma_{v_{1}^{i}}\right) P_{i}\right) \text {, } \\
& a_{i}\left(v_{1}^{j}\right) \triangleq C\left(\beta_{1} \gamma_{v_{1}^{j}} P_{j}, \beta_{1}\left(1-\gamma_{v_{1}^{j}}\right) P_{j}+\left(1-\gamma_{v_{1}^{i}}\right) P_{i}\right) \text {, } \\
& a_{i}\left(v_{2}^{i}\right) \triangleq C\left(\gamma_{v_{2}^{i}} P_{i}, \beta_{1}\left(1-\gamma_{v_{1}^{j}}\right) P_{j}+\left(1-\Gamma_{v_{2}^{i}}\right) P_{i}\right) \text {, } \\
& a_{i}\left(v_{3}^{i}\right) \triangleq C\left(\gamma_{v_{3}^{i}} P_{i}, \beta_{1}\left(1-\gamma_{v_{1}^{j}}\right) P_{j}+\Gamma_{u_{3}^{i}} P_{i}\right) \text {, } \\
& a_{i}\left(u_{1}^{i}\right) \triangleq C\left(\gamma_{u_{1}^{i}} P_{i}, \beta_{1}\left(1-\gamma_{v_{1}^{j}}\right) P_{j}+\left(\gamma_{u_{2}^{i}}+\gamma_{u_{3}^{i}}\right) P_{i}\right) \text {, } \\
& b_{i}\left(v_{1}^{i}\right) \triangleq C\left(\gamma_{v_{1}^{i}} P_{i}, \beta_{2} P_{j}+\left(1-\gamma_{v_{1}^{i}}\right) P_{i}\right) \text {, } \\
& b_{i}\left(v_{1}^{j}\right) \triangleq C\left(\beta_{2} \gamma_{v_{1}^{j}} P_{j}, \beta_{2}\left(1-\gamma_{v_{1}^{j}}\right) P_{j}+\left(1-\gamma_{v_{1}^{i}}\right) P_{i}\right) \text {, } \\
& b_{i}\left(v_{2}^{i}\right) \triangleq C\left(\gamma_{v_{2}^{i}} P_{i}, \beta_{2}\left(1-\gamma_{v_{1}^{j}}\right) P_{j}+\left(1-\Gamma_{v_{2}^{i}}\right) P_{i}\right) \text {, } \\
& b_{i}\left(v_{2}^{j}\right) \triangleq C\left(\beta_{2} \gamma_{v_{2}^{j}} P_{j}, \beta_{2}\left(1-\Gamma_{v_{2}^{j}}\right) P_{j}+\left(1-\Gamma_{v_{2}^{i}}\right) P_{i}\right) \text {, } \\
& b_{i}\left(v_{3}^{i}\right) \triangleq C\left(\gamma_{v_{3}^{i}} P_{i}, \beta_{2}\left(1-\Gamma_{v_{2}^{j}}\right) P_{j}+\Gamma_{u_{3}^{i}} P_{i}\right) \text {, } \\
& b_{i}\left(u_{1}^{i}\right) \triangleq C\left(\gamma_{u_{1}^{i}} P_{i}, \beta_{2}\left(1-\Gamma_{v_{2}^{j}}\right) P_{j}+\left(\gamma_{u_{2}^{i}}+\gamma_{u_{3}^{i}}\right) P_{i}\right) \text {, } \\
& b_{i}\left(u_{2}^{i}\right) \triangleq C\left(\gamma_{u_{2}^{i}} P_{i}, \beta_{2}\left(1-\Gamma_{v_{2}^{j}}\right) P_{j}+\gamma_{u_{3}^{i}} P_{i}\right) \text {, } \\
& c_{i}\left(v_{1}^{j}\right) \triangleq C\left(\beta_{3} \gamma_{v_{1}^{j}} P_{j}, \beta_{3}\left(1-\gamma_{v_{1}^{j}}\right) P_{j}+P_{i}\right) \text {, } \\
& c_{i}\left(v_{1}^{i}\right) \triangleq C\left(\gamma_{v_{1}^{i}} P_{i}, \beta_{3}\left(1-\gamma_{v_{1}^{j}}\right) P_{j}+\left(1-\gamma_{v_{1}^{i}}\right) P_{i}\right), \\
& c_{i}\left(v_{2}^{j}\right) \triangleq C\left(\beta_{3} \gamma_{v_{2}^{j}} P_{j}, \beta_{3}\left(1-\Gamma_{v_{2}^{j}}\right) P_{j}+\left(1-\gamma_{v_{1}^{i}}\right) P_{i}\right), \\
& c_{i}\left(v_{2}^{i}\right) \triangleq C\left(\gamma_{v_{2}^{i}} P_{i}, \beta_{3}\left(1-\Gamma_{v_{2}^{j}}\right) P_{j}+\left(1-\Gamma_{v_{2}^{i}}\right) P_{i}\right), \\
& c_{i}\left(v_{3}^{j}\right) \triangleq C\left(\beta_{3} \gamma_{v_{3}^{j}} P_{j}, \Gamma_{u_{3}^{j}} \beta_{3} P_{j}+\Gamma_{u_{3}^{i}} P_{i}\right) \text {, } \\
& c_{i}\left(v_{3}^{i}\right) \triangleq C\left(\gamma_{v_{3}^{i}} P_{i}, \Gamma_{u_{3}^{j}} \beta_{3} P_{j}+\Gamma_{u_{3}^{i}} P_{i}\right) \text {, } \\
& c_{i}\left(u_{1}^{i}\right) \triangleq C\left(\gamma_{u_{1}^{i}} P_{i}, \Gamma_{u_{3}^{j}} \beta_{3} P_{j}+\left(\gamma_{u_{2}^{i}}+\gamma_{u_{3}^{i}}\right) P_{i}\right) \text {, } \\
& c_{i}\left(u_{2}^{i}\right) \triangleq C\left(\gamma_{u_{2}^{i}} P_{i}, \Gamma_{u_{3}^{j}} \beta_{3} P_{j}+\gamma_{u_{3}^{i}} P_{i}\right) \text {, } \\
& c_{i}\left(u_{3}^{i}\right) \triangleq C\left(\gamma_{u_{3}^{i}} P_{i}, \Gamma_{u_{3}^{j}} \beta_{3} P_{j}\right) \text {. }
\end{aligned}
$$

\section{REFERENCES}

[1] T. Han and K. Kobayashi, "A new achievable rate region for the interference channel," IEEE Transactions on Information Theory, vol. 27, no. 1, pp. 49-60, Jan. 1981.

[2] H.-F. Chong, M. Motani, H. K. Garg, and H. El Gamal, "On the HanKobayashi region for the interference channel," IEEE Transactions on Information Theory, vol. 54, no. 7, pp. 3188-3195, Jun. 2008.

[3] R. H. Etkin, N. David, and H. Wang, "Gaussian interference channel capacity to within one bit," IEEE Transactions on information theory, vol. 54, no. 12, pp. 5534-5562, Nov. 2008. 
[4] A. Carleial, "A case where interference does not reduce capacity," IEEE Transactions on Information Theory, vol. 21, no. 5, pp. 569-570, Sep. 1975.

[5] H. Sato, "The capacity of the gaussian interference channel under strong interference," IEEE Transactions on Information Theory, vol. 27, no. 6 , pp. 786-788, Nov. 1981.

[6] R. Benzel, "The capacity region of a class of discrete additive degraded interference channels," IEEE Transactions on Information Theory, vol. 25, no. 2, pp. 228-231, Mar. 1979.

[7] A. Gamal and M. Costa, "The capacity region of a class of deterministic interference channels," IEEE Transactions on Information Theory, vol. 28, no. 2, pp. 343-346, Mar. 1982.

[8] V. R. Cadambe, S. A. Jafar, and S. Vishwanath, "The capacity region of a class of deterministic Z channels," in Proc. IEEE International Symposium on Information Theory, Seoul, South Korea, Jul. 2009, pp. 2634-2638.

[9] H.-F. Chong, M. Motani, and H. K. Garg, "The capacity region of a class of interference channels," in Proc. IEEE International Symposium on Information Theory, Nice, France, Jun. 2007, pp. 2856-2860.

[10] G. Bresler and D. Tse, "The two-user Gaussian interference channel: A deterministic view," European Transactions on Telecommunications, vol. 19, no. 4, pp. 333-354, Apr. 2008.

[11] M. A. Maddah-Ali, A. S. Motahari, and A. K. Khandani, "Communication over MIMO X channels: Interference alignment, decomposition, and performance analysis," IEEE Transactions on Information Theory, vol. 54, no. 8, pp. 3457-3470, Aug. 2008.

[12] V. R. Cadambe and S. A. Jafar, "Interference alignment and degrees of freedom of the $K$-user interference channel," IEEE Transactions on Information Theory, vol. 54, no. 8, pp. 3425 - 3441, Jul. 2008.

[13] T. Cover, "Broadcast channels," IEEE Transactions on Information Theory, vol. 18, no. 1, pp. 2-14, Jan. 1972.

[14] S. Shamai (Shitz), "A broadcast strategy for the Gaussian slowly fading channel," in Proc. IEEE International Symposium Information Theory, Ulm, Germany, Jun. 1997, p. 150.

[15] S. Shamai (Shitz) and A. Steiner, "A broadcast approach for a single- user slowly fading MIMO channel," IEEE Transactions on Information Theory, vol. 49, no. 10, pp. 2617-2635, Oct. 2003.

[16] S. Shamai (Shitz), "A broadcast approach for the multiple-access slow fading channel," in Proc. IEEE International Symposium Informormation Theory, Sorrento, Italy, Jun. 2000, p. 128.

[17] P. Minero and D. N. C. Tse, "A broadcast approach to multiple access with random states," in Proc. IEEE International Symposium Informormation Theory, Nice, France, Jun. 2007, pp. 2566-2570.

[18] S. Zou, Y. Liang, and S. S. (Shitz), "Multiple access channel with state uncertainty at transmitters," in Proc. IEEE International Symposium Informormation Theory, Istanbul, Turkey, Jul. 2013, pp. 1466-1470.

[19] S. Kazemi and A. Tajer, "Multiaccess communication via a broadcast approach adapted to the multiuser channel," IEEE Transactions on Communications, vol. 66, no. 8, pp. 3341-3353, Feb. 2018.

[20] M. Zohdy, S. Kazemi, and A. Tajer, "A broadcast approach to multiple access with partial CSIT," in Proc. IEEE Global Communications Conference, Abu Dhabi, UAE, Dec. 2018.

[21] M. Zohdy, A. Tajer, and S. Shamai (Shitz), "Broadcast approach to multiple access with local CSIT," IEEE Transactions on Communications, vol. 67, no. 11, pp. 7483-7498, Aug. 2019.

[22] C. Geng, N. Naderializadeh, A. S. Avestimehr, and S. A. Jafar, "On the optimality of treating interference as noise," IEEE Transactions on Information Theory, vol. 61, no. 4, pp. 1753-1767, 2015.

[23] M. Ashraphijuo, A. Tajer, C. Gong, and X. Wang, "A Receivercentric Approach to Interference Management: Fairness and Outage Optimization," in IEEE Transactions on Information Theory, vol. 62, no. 10, Oct. 2016, pp. 5619-5642.

[24] X. Yi and H. Sun, "Opportunistic treating interference as noise," IEEE Transactions on Information Theory, vol. 66, no. 1, pp. $520-533$, Jan. 2020.

[25] A. Carleial, "Interference channels," IEEE Transactions on Information Theory, vol. 24, no. 1, pp. 60-70, Jan. 1978.

[26] I. Sason, "On achievable rate regions for the Gaussian interference channel," IEEE Transactions on Information Theory, vol. 50, no. 6, pp. 1345-1356, Jun. 2004. 